\title{
Depresión y adherencia en personas afectadas con tuberculosis. Una exploración preliminar de datos.
}

\begin{abstract}
Association between depression and the lack of adherence to antituberculosis treatment.
Félix Llanos-Tejada ${ }^{1,2, a}$, César Ponce-Chang ${ }^{1,3, b}$.

RESUMEN

Objetivo: Determinar la asociación entre depresión y la falta de adherencia al tratamiento antituberculoso en pacientes del Hospital Nacional Dos de Mayo (Lima, Perú) durante el año 2017. Material y Métodos: Estudio observacional, descriptivo y prospectivo en 50 pacientes con Tuberculosis que finalizaron la primera fase del Esquema Sensible de tratamiento. Se aplicó el cuestionario PHQ-9 para determinar depresión, y el cuestionario Morisky-Green para evaluar adherencia al tratamiento. Resultados: Se evaluaron 28 pacientes varones y 22 mujeres con diagnóstico reciente de TB pulmonar y estado nutricional normal más frecuente. El promedio de faltas fue $6.5 \%$. Un 38\% de pacientes fueron clasificados como no adherentes y la depresión estuvo presente en un $76 \%$. Se determinó asociación estadísticamente significativa $(\mathrm{p}>0.05)$ entre niveles de adherencia y de depresión, según los instrumentos utilizados. Igualmente, se encontró asociación entre adherencia y tener 5 o más faltas durante la primera fase del tratamiento. Conclusiones: Existe asociación entre el nivel de adherencia al tratamiento anti-TB y la presencia de depresión. Asimismo, la elevada frecuencia de no adherencia al tratamiento en primera fase y la frecuencia elevada de síntomas sugestivos de depresión y la asociación entre adherencia y tener 5 o más faltas.
\end{abstract}

PALABRAS CLAVES: Tuberculosis, depresión, adherencia al tratamiento.

\section{SUMMARY}

Objective: To determine the association between depression and the lack of adherence to antituberculosis treatment in patients treated at the National Hospital Dos de Mayo (Lima, Perú) in 2017. Material and Methods: Observational, descriptive and prospective study carried out in $50 \mathrm{~TB}$ patients who completed the first phase of the Sensitive treatment scheme. PHQ-9 questionnaire was applied to determine depression, and Morisky-Green questionnaire to evaluate adherence to treatment. Results: 28 male and 22 female patients with normal nutritional status, recently diagnosed with pulmonary TB were assessed. Average number of fouls was $6.5 .38 \%$ of patients were non-adherent, and depression was present in $76 \%$, according to the instruments used. ( $>0.05)$. There was also a statistically significant association between adherence and having 5 or more faults during the 1st Phase. Conclusions: There is an association between adherence to anti-TB treatment and depression. Similarly, high frequency of non-adherence to treatment in the first phase and high frequency of symptoms suggestive of depression, and association between adherence and having 5 or more faults.

KEYWORDS: Tuberculosis, depression, patient adherence.

Facultad de Medicina, Universidad San Martín de Porres. Lima, Perú.

Hospital Nacional Dos de Mayo. Lima, Perú.

Hospital Nacional Daniel Alcides Carrión. Callao, Perú.

Médico Neumólogo; ${ }^{\mathrm{b}}$ Interno de Medicina. 
Depresión y adherencia en personas afectadas con tuberculosis. Una exploración preliminar de datos.

\section{INTRODUCCIÓN}

La tuberculosis (TB) es un problema de salud pública a nivel mundial. En el año 2013, César Ugarte realizó un trabajo en el que demostró que el $37 \%$ de los pacientes con TB presentaban un trastorno depresivo mayor y que este se asociaba a resultados negativos en el tratamiento antituberculoso. Sin embargo, en dicho estudio no se determinó si existe una asociación directa entre la depresión y la falta de adherencia al tratamiento antituberculoso (1).

La falta de adherencia al tratamiento antituberculoso se relaciona con un riesgo incrementado de persistencia de transmisión de la enfermedad a la población, incremento de la resistencia, aumento de la mortalidad y disminución de la calidad de vida.

Existen diversos estudios, en los que se determina la relación e influencia de la depresión en la evolución de algunas enfermedades crónicas, tal como lo refiere Wayne Katon en su estudio "La depresión y la diabetes: una combinación potencialmente fatal", donde pudo demostrar que la depresión en personas con diabetes está relacionado a una baja adherencia a los regímenes de cuidado personal, con un mayor riesgo de complicaciones microvasculares y macrovasculares y un aumento del riesgo de la mortalidad a los 2 años (2).

La depresión puede establecerse mediante evaluaciones como la Escala de Depresión PHQ-9 (Patient Health Questionnaire), que es un test que consta de 9 ítems que evalúan la presencia de síntomas depresivos, correspondientes a los criterios DSM-IV, presentes en las dos últimas semanas $(3,4)$.

Asimismo, la adherencia o cumplimiento al tratamiento de un paciente puede valorarse mediante métodos como la Prueba de Morisky-Green, el mismo que está validado en diferentes enfermedades crónicas $(5,6)$.

Consideramos que esta investigación es innovadora, pues tiene como objetivo determinar la asociación entre la depresión, medida por PHQ-9, y la falta de adherencia al tratamiento antituberculoso, medido por Morisky-Green, en pacientes atendidos en el Hospital Nacional Dos de Mayo durante el año 2017.

\section{MATERIAL Y MÉTODOS}

El presente es un estudio cuantitativo, de tipo descriptivo, transversal y prospectivo.

Se incluyeron a los pacientes con TB en tratamiento Sensible (Esquema Uno), que hayan culminado la Primera Fase del tratamiento. Se consideró como criterio de exclusión la no intención de querer participar firmando el consentimiento informado. Se requirió un número de muestra de 50 participantes, los que se consiguieron mediante muestreo por conveniencia, de acuerdo a la asistencia a consulta en los establecimientos de salud.

Previamente, se capacitó a las Licenciadas de Enfermería de los establecimientos de salud San Luis y San Cosme de la jurisdicción del Hospital Nacional Dos de Mayo para realizar la escala de Depresión PHQ-9 y la prueba de Morisky-Green, a los pacientes incluidos previa firma del Consentimiento Informado. La escala PHQ-9 es una herramienta útil para establecer un diagnóstico de depresión, para ayudar en la decisión de iniciar tratamiento específico y monitorizar su tratamiento; y es auto-administrada. Se define trastorno depresivo si hay por lo menos 5 ítems puntuados al menos como "más de la mitad de los días" o "casi todos los días"; y al menos uno de los ítems positivos corresponde a la pregunta 1 o 2. Asimismo, el trastorno depresivo subumbral (Depresión menor): Si hay por lo menos 2 a 4 ítems puntuados al menos como "más de la mitad de los días" o "casi todos los días"; y al menos uno de los ítems positivos corresponde a la pregunta 1 o 2 .

La prueba de Morisky-Green es uno de los métodos más utilizados para evaluar si un paciente cumple con el tratamiento pautado, que aunque sencillo, es suficiente y aplicable a todos los pacientes. Consiste en la aplicación al paciente de estas 4 preguntas, y en todos los casos se debe responder "SI" o "NO". Se consideran pacientes que cumplen el tratamiento a quienes contestan NO a las cuatro preguntas y no cumplidores a quienes contestan SI a una o más. Es muy eficaz, especialmente en enfermedades crónicas como hipertensión arterial y diabetes mellitus 2 .

Se evaluaron las frecuencias de depresión y el promedio del puntaje en la escala PHQ-9, tanto en el grupo con mala adherencia, como de buena adherencia al tratamiento, de acuerdo a la prueba de MoriskyGreen. Se elaboraran tablas de 2x2 para determinar el Chi cuadrado y estimar el riesgo de mala adherencia y 
depresión. Los datos fueron procesados en el paquete estadístico SPSS.

Este trabajo fue revisado por el Comité de Ética de la Facultad de Medicina de la Universidad San Martin de Porres y del Hospital Nacional Dos de Mayo.

\section{RESULTADOS}

Se evaluaron 50 pacientes que aceptaron participar en el estudio y cuyos datos fueron recolectados en los establecimientos de salud de la jurisdicción del Hospital Nacional Dos de Mayo.

La edad promedio fue $35+/-12,5$ años. De los 50 pacientes, 28 fueron del sexo masculino (56\%). En el estado nutricional, predominó el grupo con peso normal con una frecuencia de 33 pacientes $(66 \%)$ (tabla 1).

Según la localización de la Tuberculosis (TB), 42 pacientes presentaron TB pulmonar $(84 \%)$ y 8 pacientes presentaron TB extrapulmonar. La condición de ingreso más frecuente fue Nuevo, con 38 pacientes (76\%) (tabla 1).

El número promedio de faltas que presentó un paciente en primera Fase fue 6,5\%, con un máximo de 37 faltas registradas. De acuerdo a la prueba de Morisky-Green, 19 de los pacientes eran no adherentes

Tabla 1. Características demográficas.

\begin{tabular}{lcc}
\hline & $\mathrm{n}$ & $\%$ \\
\hline TOTAL & $\mathbf{5 0}$ & $\mathbf{1 0 0}$ \\
Sexo & & \\
Masculino & 28 & $56,00 \%$ \\
Femenino & 22 & $44,00 \%$ \\
Estado Nutricional & & \\
Bajo peso & 3 & $6,00 \%$ \\
Peso normal & 33 & $66,00 \%$ \\
Sobrepeso & 12 & $24,00 \%$ \\
Obesidad & 2 & $4,00 \%$ \\
Condición de Ingreso & & \\
Nuevo & 38 & $76,00 \%$ \\
Abandono recuperado & 6 & $12,00 \%$ \\
Recaída & 6 & $12,00 \%$ \\
\hline
\end{tabular}

Tabla 2. Distribución de depresión y adherencia.

\begin{tabular}{lcc}
\hline PHQ-9 & n & $\mathbf{\%}$ \\
\hline Síndrome depresivo mayor (SDM) & 3 & $6,00 \%$ \\
Otros síndromes depresivos (OSD) & 5 & $10,00 \%$ \\
Síntomas depresivos positivos (SDP) & 30 & $60,00 \%$ \\
Síntomas depresivos negativos (SDN) & 12 & $24,0 \%$ \\
Morisky-Green & & \\
Cumplidor/Adherente & 31 & $62,0 \%$ \\
No cumplidor/No Adherente & 19 & $38,0 \%$ \\
\hline
\end{tabular}

Tabla 3. Evaluación adherencia con depresión y número de faltas

\begin{tabular}{cccc}
\hline \multicolumn{3}{c}{ Test de Morisky Green } \\
& Adherente & No adherente & \\
\hline 3 o más & $35,48 \%$ & $57,89 \%$ & $\mathrm{p}>0,05$ \\
FALTAS & & & \\
5 o más & $29,03 \%$ & $68,42 \%$ & $\mathrm{p}<0,05$ \\
FALTAS & & $36,84 \%$ & \\
Depresión (+) & $63,16 \%$ & $\mathrm{P}<0,05$ \\
Depresión (-) & $51,33 \%$ & $48,67 \%$ & \\
\hline
\end{tabular}

al tratamiento (38\%),y se determinó depresión en 38 pacientes, 3 de ellos con Síndrome Depresivo Mayor (tabla 2).

Pudimos determinar asociación estadísticamente significativa entre nivel de adherencia según MoriskyGreen y depresión según PHQ-9 $(\mathrm{p}<0,05)$. Se pudo determinar una asociación entre Morisky-Green y tener 5 o más faltas durante la primera fase (tabla 3 ).

\section{DISCUSIÓN}

En el presente estudio hemos podido demostrar una asociación entre la depresión y la falta de adherencia al tratamiento antituberculoso en los pacientes evaluados, a los cuales se les aplicó la prueba de Morisky-Green y la escala de depresión PHQ-9.

En la presente investigación se encontró una media de edad de 34 años, siendo más frecuente el sexo masculino (56\%). Esto coincide con otros estudios previos $(1,7,8)$, y las estadísticas del Ministerio de Salud (MINSA), que reportan que la TB es más frecuente en población económicamente activa (PEA) masculina. 
Depresión y adherencia en personas afectadas con tuberculosis. Una exploración preliminar de datos.

El estado nutricional de normalidad fue el más frecuente, contrario a lo que considera el público general, que la TB es más frecuente en desnutrición. Nuestros resultados son similares a lo reportado en un estudio previo, donde la mayor parte de los pacientes con el diagnostico de TB tenían un peso normal. Y, en dicho estudio se afirma que los pacientes que reciben tratamiento antituberculoso presentan tendencia a favorecer la migración de su estado nutricional a una categoría superior por diversas causas, entre ellas el aumento de apetito (9).

En nuestro estudio se encontró una prevalencia de $16 \%$ de TB extrapulmonar, sin que podamos encontrar que estos pacientes tengan una mejor o peor adherencia que los pacientes con TB pulmonar. Estos datos son similares a la estadística mostrada por otros autores, en los cuales se afirma que la TB extrapulmonar representa entre un 10 a $20 \%$ de la población con esta enfermedad (10). Recalcamos que una mala adherencia al tratamiento antituberculoso es sumamente importante en TB extrapulmonar ya que aumentar la sintomatología clínica del paciente y, potencialmente, disminuir la calidad de vida $(11,12)$.

Con respecto a la condición de ingreso, en nuestro estudio la mayoría de pacientes fueron nunca tratados. Es importante mencionar que aquellos pacientes antes tratados, que tuvieron recaída o ingresaron como abandonos recuperados, tuvieron peor adherencia al tratamiento antituberculoso y algún grado de depresión. Es un dato muy importante debido a que otros autores refieren que los pacientes que presentan el antecedente de abandono del tratamiento antituberculoso u otro tratamiento, tienen riesgo muy elevado de volver a abandonar el mismo $(7,12,13)$; sin embargo, nosotros no pudimos demostrar una diferencia estadísticamente significativa, quizás debido al tamaño de muestra.

La norma técnica de salud para la atención integral de las personas afectadas por tuberculosis define la irregularidad del tratamiento como el tener más de 3 faltas durante la primera fase, o más de 5 faltas durante todo el tratamiento antituberculoso (14), por lo que podemos considerar que la media de faltas durante la primera fase del esquema sensible en nuestro estudio es alta. Estas estadísticas muestran el agravamiento del problema de salud pública que representa la TB en nuestro país. Según lo señalado Zhang et al., la mala adherencia al tratamiento antituberculoso implica una persistencia de la fuente de infección, un aumento de la mortalidad y de la tasa de resistencia; además, puede facilitar la aparición de cepas de bacilos resistentes
(15). Por lo que sería bueno aplicar otras estrategias aparte de las ya existentes, para disminuir el número de faltas o mejorar la adherencia.

Hemos podido determinar una frecuencia de depresión del 76\%, siendo el Síndrome Depresivo Mayor (SDM) el 6\% de la misma. Ugarte-Gil realizó un estudio donde encontró que el $37 \%$ de la población con el diagnostico de TB presentó un SDM, por lo que en nuestro estudio la prevalencia del mismo se encuentra por debajo de lo esperado (1). Sin embargo, la prevalencia de depresión en nuestros pacientes equivale a tres veces más de lo esperado a las estadísticas mostradas por otros autores con respecto a la prevalencia de la misma para Lima Metropolitana (16). Las enfermedades mentales son causa de sufrimiento personal para quienes la padecen y para las personas de su entorno. Una revisión de Argiro Pachi y colaboradores enfatiza el impacto de las enfermedades mentales, especialmente la depresión y la ansiedad, en la adherencia al tratamiento antituberculosis y la interacción entre fármacos (17). El Perú es un país donde la atención psiquiatría no está disponible en varias regiones y donde los seguros no están obligados por ley a cubrir tales atenciones (16). Las enfermedades mentales son causa de discapacidad y detención del desarrollo, por lo que sería bueno, dada la alta prevalencia de depresión en los pacientes con $\mathrm{TB}$, implementar atención psicológica y/o psiquiátrica para los mismos.

Nuestros resultados indican que el $38 \%$ de los pacientes con TB fueron no adherentes a su tratamiento. El método que se uso fue el test de Morisky Green, ya validado y aplicado en numerosas patologías, ampliamente conocido por su alto valor predictivo positivo $(5,6)$. Según lo reportado por otros autores, la adherencia terapéutica es un fenómeno multidimensional determinado por la acción reciproca de cinco conjuntos de factores, entre los cuales se encuentran: factores socioeconómicos, factores relacionados con el equipo o sistema de asistencia sanitaria, factores relacionados con la enfermedad, factores relacionados con el tratamiento y factores relacionados con el paciente (18). Esta alta prevalencia de pacientes no adherentes al tratamiento antituberculoso representan un gran problema en la salud pública para el país.

Anduaga et al., pudieron determinar que el cuestionario de predicción de abandono al tratamiento anti-tuberculosis, que incluye una prueba rápida de depresión, similar al Yesavage abreviado, está 
asociado al abandono si se obtienen más de 22 puntos (19).

Otros estudios nacionales, han valorado evaluar los factores de riesgo para el abandono, tal como Anduaga (19) que lo asoció a nivel educativo y Lackey et al., a nivel educativo, farmacodependencia y alcoholismo (20).

En el presente estudio se buscó como objetivo secundario determinar la asociación entre el tener más de 3 faltas al tratamiento antituberculoso durante la primera fase de acuerdo a la Norma técnica de atención integral de las personas con tuberculosis que rige el MINSA, y el ser adherente o no adherente de acuerdo al test de Morisky Green; al no poder demostrar esta asociación, nos hace inferir que el tener 3 faltas o más durante la primera fase del tratamiento antituberculoso no es suficiente para considerarse adherente o no adherente al tratamiento del mismo.

Así, al evaluar nuestros aciertos y desaciertos en torno a la TB como problema de salud pública en nuestro medio, logramos determinar asociación entre presentar 5 o más faltas durante la primera fase del tratamiento antituberculoso y el ser adherente o no, de acuerdo al test de Morisky Green. Somos conscientes de los efectos negativos que conllevan el ser no adherente al tratamiento antituberculoso, expuestos anteriormente. Sin embargo, no basta con tener la definición de adherencia, sino también, de la terminología empleada a su alrededor, entre ellas, el saber desde cuándo se puede considerar a un paciente regular o irregular al tratamiento antituberculoso. Sería beneficioso el considerar reevaluar la definición de irregularidad del tratamiento según la Norma técnica de atención integral de las personas con tuberculosis, considerando que nuestros resultados sugieren que 5 faltas durante la primera fase se asocia con la prueba predictiva del Morisky-Green.

En conclusión, podemos afirmar que el presente estudio pudo determinar la asociación entre la depresión y la falta de adherencia al tratamiento antituberculoso medido por el Test de Morisky-Green, y que esta prueba se relaciona con la inasistencia a por lo menos 5 tomas durante la primera fase del esquema sensible.

Asimismo, podemos afirmar que aproximadamente tres cuartas partes de los pacientes con el diagnostico de TB sufrían algún grado de depresión.
Recomendamos realizar un estudio similar con un mayor número de muestra o realizar un estudio de tipo analítico.

Asimismo, se recomienda brindar atención psicología y/o psiquiátrica a los pacientes con el diagnostico de TB debido a la alta prevalencia de depresión que presentan y al posible riesgo de mala adherencia al tratamiento, tal como lo han hecho en otros países con experiencias exitosas (21).

\section{Correspondencia}

Félix Llanos-Tejada

Servicio de Neumología, Hospital Nacional Dos de Mayo.

Parque Historia de la Medicina s/n. Cercado de Lima. Lima, Perú.

Correo electrónico: neumofekollate@aol.com // fllanost@usmp.pe

Contribuciones de los Autores: CPC y FLT participaron en la concepción y diseño del trabajo, recolección de datos, interpretación de datos, redacción y revisión crítica del manuscrito y aprobación de su versión final. FLT participó, además, en la asesoría estadística y asesoría técnica.

Fuente de Financiamiento: Estudio Auto-financiado.

Los autores declaran no tener conflicto de intereses relacionado directa indirectamente al presente estudio de investigación.

\section{REFERENCIAS BIBLIOGRÁFICAS}

1. Ugarte-Gil C, Ruiz P, Zamudio C, Canaza L, Otero L, Kruger $\mathrm{H}$, et al. Association of major depressive episode with negative outcomes of tuberculosis treatment. PLoS ONE. 2013; 8(7): e69514. DOI: https://doi.org/10.1371/journal.pone.0069514

2. Katon W, Fan M-Y, Unützer J, Taylor J, Pincus H, Schoenbaum M. Depression and diabetes: a potentially lethal combination. J Gen Intern Med. 2008; 23(10):1571-5.

3. Baader T, Molina JL, Venezian S, Rojas C, Farías $\mathrm{R}$, Fierro-Freixeneta $\mathrm{C}$, et al. Validación y utilidad de la encuesta PHQ-9 (Patient Health Questionnaire) en el diagnóstico de depresión en pacientes usuarios de atención primaria en Chile. Rev. Chil NeuroPsiquiatr. 2012; 50(1): 10-22.

4. Kroenke K, Spitzer RL, Williams JBW. The PHQ-9: Validity of a brief depression severity measure. Journal of General Internal Medicine. 2001; 16 (9): 606-613. 
Depresión y adherencia en personas afectadas con tuberculosis. Una exploración preliminar de datos.

5. Rodríguez M, García-Jiménez E, Amariles P, Rodríguez A, Faus M. Revisión de tests de medición del cumplimiento terapéutico utilizados en la práctica clínica. Aten Primaria. 2008; 40: 413-8.

6. Limaylla M, Ramos N. Métodos indirectos de valoración del cumplimiento terapéutico. Ciencia e Investigación. 2016; 19(2): 95-101.

7. Bernabé A. Detección temprana de fracasos a tratamiento en pacientes con tuberculosis pulmonar. Rev Med Hered. 2007; 18(3): 123-128. DOI: https:// doi.org/10.20453/rmh.v18i3.919

8. Arriola-Huerta P, Castillo-Cahuana T, Quispe-Fabián G,Torres-Deza C. Factores asociados a la asistencia del paciente al tratamiento antituberculoso. Rev enferm Herediana. 2011;4(2):86-92.

9. Aparco JP, Huamán-Espino L, Segura ER. Variación del estado nutricional durante el tratamiento antituberculoso en beneficiarios del programa PANTBC. Rev Peru Med Exp Salud Publica. 2012;29(3):324-8.

10. Fanlo P, Tiberio G. Tuberculosis extrapulmonar. Anales Sis San Navarra. 2007; 30 (Suppl 2): 143162.

11. Arciniegas W, Orjuela DL. Tuberculosis extrapulmonar: revisión de 102 casos en el Hospital Universitario San Jorge de Pereira, 2000-2004. Biomedica. 2006; 26(1): 70-80.

12. Torres Z, Herrera T. Perfil del paciente con tuberculosis que abandona el tratamiento en chile Rev Chil Enf Respir 2015; 31: 52-57.

13. Culqui DR, Munayco E. CV, Grijalva CG, Cayla JA, Horna-Campos O, Alva K, et al. Factores asociados al abandono de tratamiento antituberculoso convencional en Perú. Archivos deBronconeumología. 2012;48(5):150-5.

14. Ministerio de Salud. Norma técnica de salud para la atención integral de las personas afectadas por tuberculosis. Lima: Ministerio de Salud; 2013.

15. Zhang H, Ehiri J, Yang H, Tang S, Li Y. Impact of Community-Based DOT on Tuberculosis Treatment
Outcomes: A Systematic Review and Meta-analysis. PLoS One. 2016; 11(2):e0147744. doi: 10.1371/ journal.pone. 0147744

16. Rondón MB. Salud mental: un problema de salud pública en el Perú. Rev. Perú Med Exp Salud Pública. 2006; 23(4):237-8.

17. Pachi A, Bratis D, Moussas G, Tselebis A. Psychiatric morbidity and other factors affecting treatment adherence in pulmonary tuberculosis patients. Tuberculosis Research and Treatment. 2013;2013: 489865. Doi: https://doi.org/10.1155/2013/489865

18. Rodríguez A, Mondragón C. Adherencia a la terapia farmacológica y sus factores determinantes en pacientes con tuberculosis de un centro de salud de Santiago de Cali. Rev Colomb Cienc Quim Farm. 2014; 43(1): 104-119.

19. Anduaga-Beramendi A, Maticorena-Quevedo J, Beas R, Chanamé-Baca DM, Veramendi M, Wiegering-Rospigliosi A, et al. Factores de riesgo para el abandono del tratamiento de tuberculosis pulmonar sensible en un establecimiento de salud de atención primaria, Lima, Perú. Acta Med Peru. 2016; 33(1): 21-8.

20. Lackey B, Seas C, Van der Stuyft P, Otero L. Patient Characteristics Associated with Tuberculosis Treatment Default: A Cohort Study in a HighIncidence Area of Lima, Peru. PLoS ONE. 2015; 10(6): e0128541. doi:10.1371/journal.pone. 0128541

21. Tola HH, Shojaeizadeh D, Tol A, Garmaroudi G, Yekaninejad MS, Kebede A, et al. Psychological and educational intervention to improve tuberculosis treatment adherence in Ethiopia Based on Health Belief Model: A Cluster Randomized Control Trial. PLoS ONE. 2016; 11(5): e0155147. doi:10.1371/ journal.pone. 0155147

Recibido: 04/08/2018

Aceptado: 25/03/2019 